\title{
Inhomogeneous source uniformization using a shell mixer Köhler integrator
}

\author{
J.Chaves $^{\mathrm{a}}$, A.Cvetkovic ${ }^{\mathrm{a}, 1}$, R.Mohedano ${ }^{\mathrm{a}}$, O.Dross ${ }^{\mathrm{b}}$, M.Hernandez ${ }^{\mathrm{a}}$, P.Benitez ${ }^{\mathrm{c}, \mathrm{d}}$, J.C. Miñano ${ }^{\mathrm{c}, \mathrm{d}}$, \\ J.Vilaplana ${ }^{\mathrm{a}}$ \\ ${ }^{a}$ LPI-Europe, Edificio CEDINT, UPM Campus de Montegancedo, 28223 Pozuelo de Alarcon, \\ Madrid, Spain; ' $\mathrm{P}$ Philips Research, Mathildelaan 1, Eindhoven, The Netherlands; ${ }^{\mathrm{c}}$ Universidad \\ Politécnica de Madrid, CEDINT, UPM Campus de Montegancedo, 28223 Pozuelo de Alarcon, \\ Madrid, Spain; ${ }^{\mathrm{d}}$ LPI, 2400 Lincoln Avenue, Altadena, CA 91001, USA
}

\begin{abstract}
High flux and high CRI may be achieved by combining different chips and/or phosphors. This, however, results in inhomogeneous sources that, when combined with collimating optics, typically produce patterns with undesired artifacts. These may be a combination of spatial, angular or color non-uniformities. In order to avoid these effects, there is a need to mix the light source, both spatially and angularly. Diffusers can achieve this effect, but they also increase the etendue (and reduce the brightness) of the resulting source, leading to optical systems of increased size and wider emission angles.

The shell mixer is an optic comprised of many lenses on a shell covering the source. These lenses perform Kohler integration to mix the emitted light, both spatially and angularly. Placing it on top of a multi-chip Lambertian light source, the result is a highly homogeneous virtual source (i.e, spatially and angularly mixed), also Lambertian, which is located in the same position with essentially the same size (so the average brightness is not increased). This virtual light source can then be collimated using another optic, resulting in a homogeneous pattern without color separation.
\end{abstract}

Experimental measurements have shown optical efficiency of the shell of $94 \%$, and highly homogeneous angular intensity distribution of collimated beams, in good agreement with the ray-tracing simulations.

Keywords: General illumination, color mixing, Kohler integration

\section{INTRODUCTION}

High flux LEDs with high color rendering indices (CRI) are often composed of several chips of different colors (for example, combinations of red, green, green, blue chips). When combined with collimating optics, these LEDs result in luminaires that often produce undesirable artifacts, which may include color shadows, color fringes, variation of the white color temperature across the illuminance distribution, intensity artifacts or multiple shadows (from the different chips in the LED package), as shown in Figure 1.

It is highly desirable to have a "universal light engine" that "replaces" the non-uniform light source with a uniform "virtual" light source. Ideally, this should be a "primary" optic on top of the LED compatible with different types of secondary optics and applications. This imposes a constraint on the optic that should be small and close to the LED. Also, the resulting light source should have approximately the same size and position as the original source, but should be uniform, both spatially (no hot spots) and in color. This would make the resulting light engine useable in different luminaries and, therefore, quite flexible.

A possible way to mix the light from the different chips into a uniform source is to use diffusers on top of the LEDs. By scattering light, these mix the emissions of the different chips creating a uniform output. In particular, diffusers eliminate undesirable color artifacts. However, this comes at a cost. The efficiency of the resulting light engine will likely suffer since a considerable amount of light will be sent back and recycled through the LED package. Also, the diffusers will be the new apparent light source, which is displaced relative to the position of the original LED. The etendue of the

\footnotetext{
1 acvetkovic@lpi-europe.com
} 
resulting source will also be larger, which means that, in order to control the light, larger secondary optics will be needed. Therefore, when using diffusers, new and larger secondary optics would have to be used to achieve the same control of the light output, while eliminating artifacts.

Other options could include mixing rods, but these need to be long to properly mix the light, are complex to assemble and displace the light source relative to its original position.

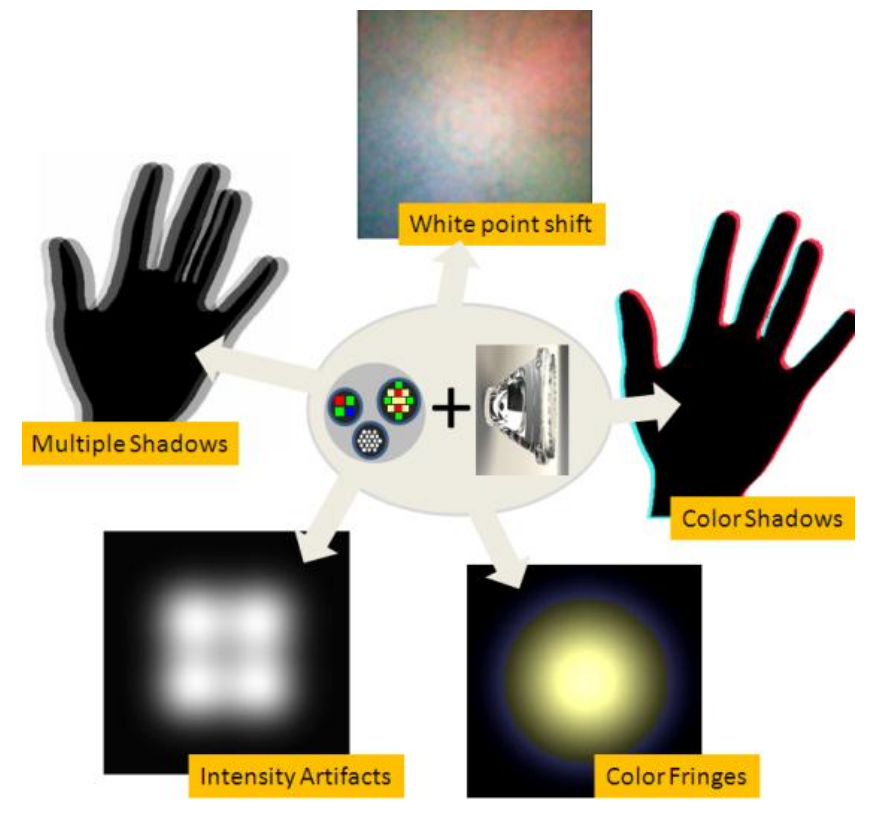

Figure 1 - Unwanted effects when using multi-chip (and especially multi-color) packages into typical luminaries.

A more practical approach is a LPI-patented solution based on the Köhler concept [2][3][4], an "integration" approach which is compatible with many different optical applications. Köhler illumination optics elements may be composed of two optical surfaces. The first surface images the source onto the second optical surface. This ensures that the light emitted from the source and falling on the first surface is redirected to the second surface. This is important for guaranteeing that no light is lost and that the Köhler element has high efficiency. The second optical surface images the first surface onto the target (or the far field). If the first surface is some distance away from the source, it will be uniformly illuminated by it. For example, if the source is a red, green, blue LED, the red, green and blue irradiances on the first surface produced by the source will be similar. By imaging this uniform irradiance onto the target, the second surface creates a uniform output pattern.

Figure 2 shows canonical example of a Köhler array composed of several elements placed side by side[5]. In this example, the source is an infinitely large emitter placed to the left and the target is also infinite, placed to the right. Both, source and target, subtend an angular aperture $\pm \alpha$ when seen from the optic. Figure 2 a shows a set of parallel rays coming from the center of the infinite source (placed to the left) coming towards the optic. Each first surface focuses (images) these rays onto the corresponding second surface. The second surface, images the first surface onto the infinite receiver, placed to the right. This means that a ray coming from the top edge of the first lens will be imaged to the bottom edge of the receiver (at an angle $\alpha$ down) and a ray coming from the bottom edge of the first lens will be imaged to the top edge of the receiver (at an angle $\alpha$ up). The result is an output spanning an angular aperture $\pm \alpha$ which fully illuminates the target. Therefore, the light coming from one point on the source to the left (its center in this case) fully illuminates the target.

Something similar may be observed in Figure $2 b$. This figure now shows a set of parallel rays coming from the top edge of the infinite source, to the left, coming towards the optic. The first set of surfaces focuses (images) these rays onto the bottom edge of the second surfaces. The second surfaces, image the first surfaces onto the infinite receiver to the right. This means that a ray coming from the top edge of the first lens will again be imaged to the bottom edge of the receiver (at an angle $\alpha$ down) and a ray coming from the bottom edge of the first lens will again be imaged to the top edge of the receiver (at an angle $\alpha$ up). The result is again an output spanning an angular aperture $\pm \alpha$ which fully illuminates the 
target. Therefore, the light coming from another point on the source to the left (its top edge in this case) still fully illuminates the target.

It may then be seen that the light coming from different points on the source produces the same output pattern coming out of the optic and, therefore, the same illuminance on the target. Also, the structure of the source is not transferred to the illuminance pattern.

There may be some light coming from outside the source, as shown in Figure 2c. In that case, the first optical surface images this light to a point outside the corresponding second optical surface and in another Köhler array element, which disperses the light outside the target.

These integrator optics may be used to homogenize the illuminance of a target, but also to create special features in the pattern, such as sharp cut offs or gradients.

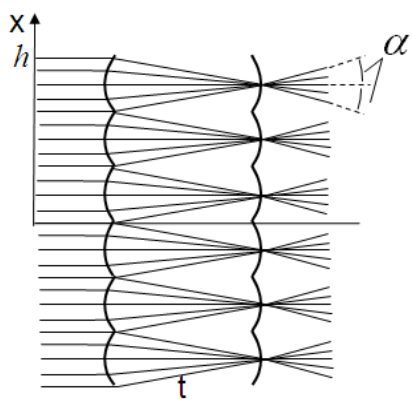

(a)

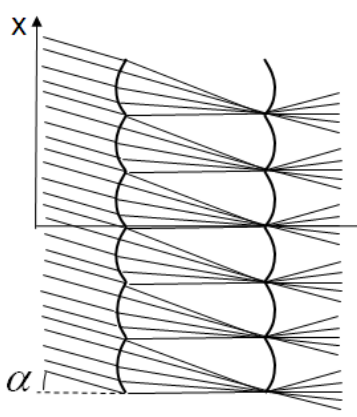

(b)

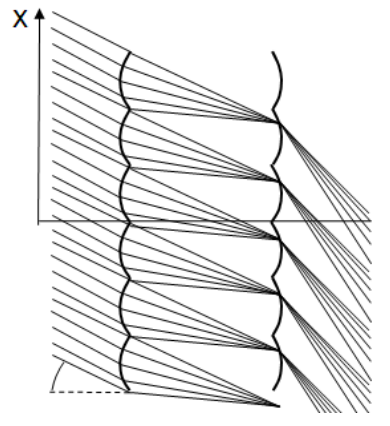

(c)

Figure 2 - Working principle of a basic integrator lens array for perpendicular (left), max angle $\alpha$ incidence (center) and illumination outside the integration zone (right)

Figure 3 shows an example of how a Kohler integrator may be used to produce white light from a red, green, blue source. On the left, this figure shows three chips of different colors. The light coming from the blue middle chip is focused to the center of the output lens and spreads out towards the target as it leaves this optical surface. In threedimensional geometry, these lenses may have square cross section. Since the second lens images the first lens onto the target, the result will be a blue square on the target. Similarly, the light coming from the red top chip is focused to the bottom of the output lens and spreads out towards the target as it leaves this optical surface. Again, the second lens images the first lens onto the target, resulting in a red square on the target. The same happens for the green chip at the bottom. The superposition of all these images (red, green and blue) is a white square, as shown on the right of Figure 3.

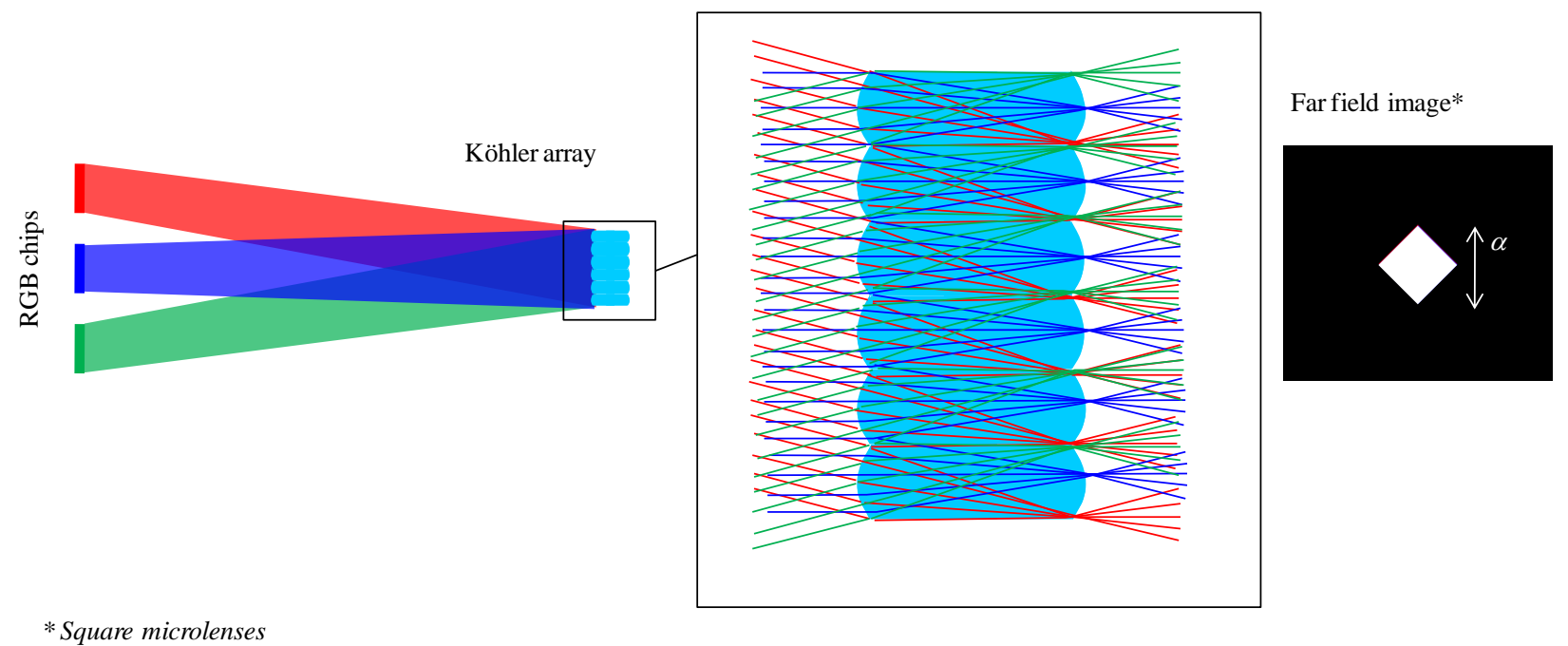

Figure 3 - Working principle of a basic integrator lens array: color mixing 


\section{OPTICAL DESIGN}

Köhler integration is not limited to flat lenslet arrays. It can be used on curved surfaces. Figure 4 shows one of these applications: the shell mixer. The optic is shaped as a dome covering the LED light source. This dome is composed of many Kohler channels, each one of these with a first optical surface that images the light source onto the second optical surface. Now, however, the second optical surface does not image the first optical surface onto the target. Instead, it generates a virtual image of the first surface that superimposes on the LED. Therefore, when seen from some distance away, the colored LED with the shell mixer on top now appears as a white uniform light source at the same position as the LED.
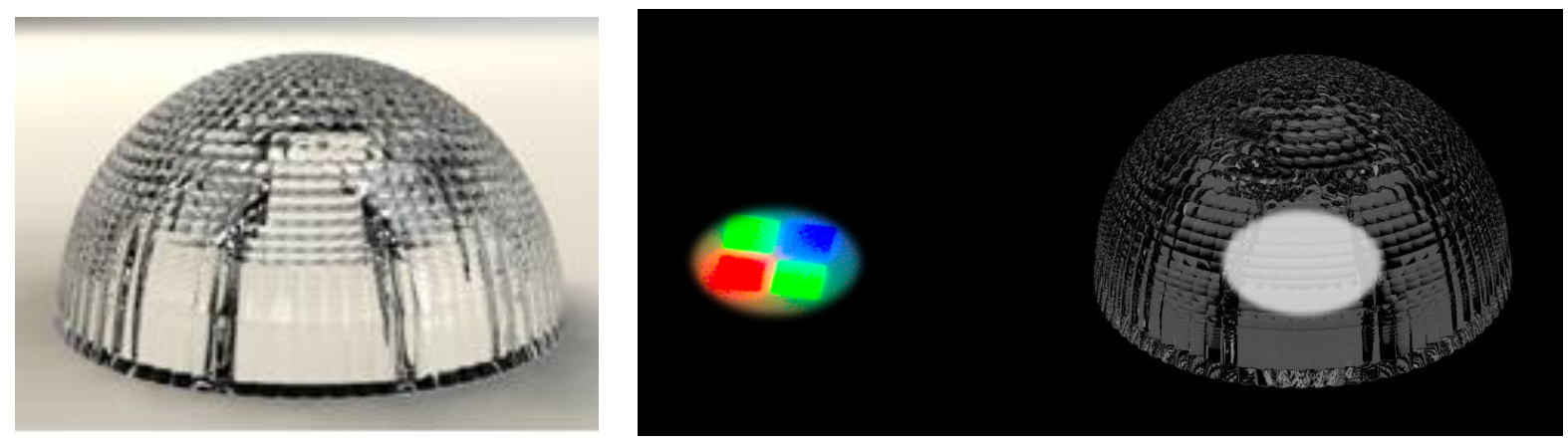

Figure 4 - Left: rendered view of the shell mixer. Right: bare colored LED and equivalent white virtual light source produced by the integration effect.

The resulting light source still emits into a hemisphere, just like the original LED. The light may now be collimated by a secondary optic, just as in the case of the bare LED. However, instead of "seeing" the LED, this secondary optic now "sees" the uniform virtual source. This drastically reduces the chances of artefacts showing up in the pattern.

Figure 5 shows a cross section of the shell mixer and the working principle of its Kohler channels (or elements). The light from colored chips at the edges of the light source is imaged by the first lens to the edges of the output lens, spreading out of the optic, as if coming from the light source. Each inner optical surface forms a channel together with an outer optical surface. The complete shell mixer is made of many of these channels side by side in a hemisphere.

In each Kohler channel, the first lens images the LED light source onto the second lens. Figure 5 on the right shows a photograph of those images formed on the output lenses.

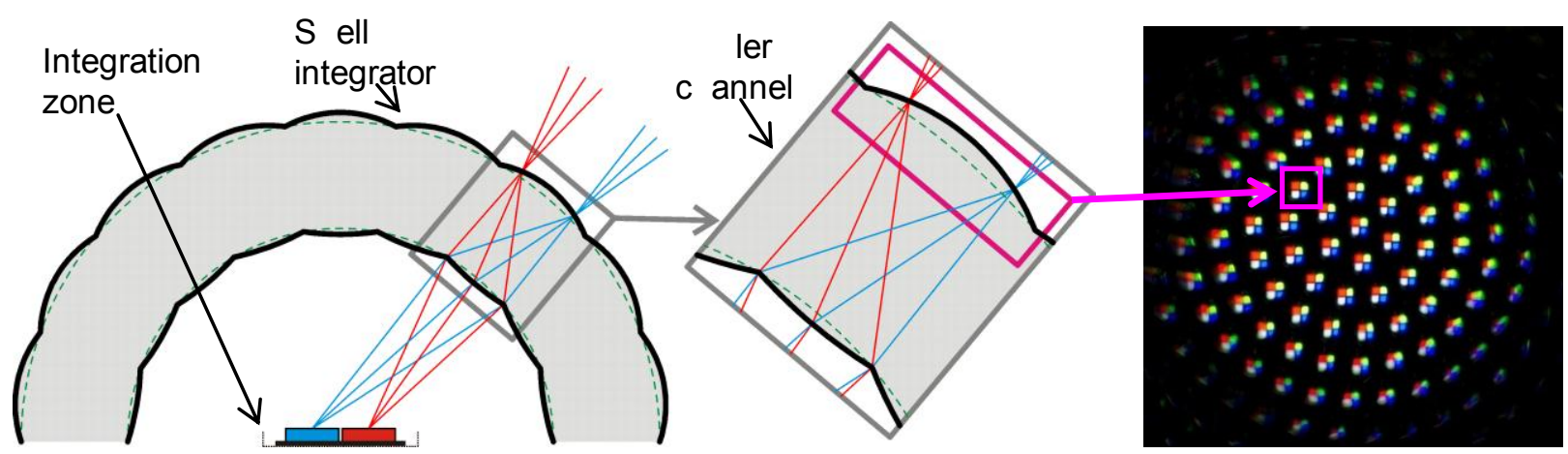

Figure 5 - Köhler channels in the shell mixer. The picture on the right shows the images of the LED produced by the inner lenses on the outer lenses of the shell mixer. 
When designing one of these optics there are several challenges that must be met. First of all, the shell mixer optic must be small, since it is supposed to be part of the light source itself. This, however, means that each lens on the inner surface of the mixer sees a wide angle source, which may be hard to image properly onto the corresponding lens on the outer surface of the mixer. Also, lenses at different positions on the mixer "see" sources with different angular apertures, which means that the Kohler channels must be different from point to point on the optic. The shell mixer should also be compatible with injection moulding manufacturing, since this is a low cost and fast way to produce these optics. This poses challenges in the design of the channels further down on the mixer (close to its rim), since the corresponding lenses (either on the inside or the outside) must have wide enough draft angles. Finally, all channels must be tessellated in a tree-dimensional optic and must work well together.

While designing and adjusting the parameters of these optics we have found that the shell mixer should have about three times the diameter of the apparent LED source if a good output with no artefacts is desired. A possible way to test for artefacts is to combine the LED and shell with an imaging lens. This large lens will image the virtual source onto a target. If there are artefacts in the virtual image generated by the shell mixer, these will show up in the image produced by this large lens. This is a worst case scenario and the mixer as shown good performance, even in these extreme conditions.

Figure 6 shows a selected optic which shows good performance when placed on a non-uniform light source. Its size may be scaled up or down to match different size sources. This optic consists of a thin shell with a thickness about $7 \%$ of its diameter. The sizes of its lenses average half of the optic's thickness, for the lenses on both the inner and outer surfaces of the optic.
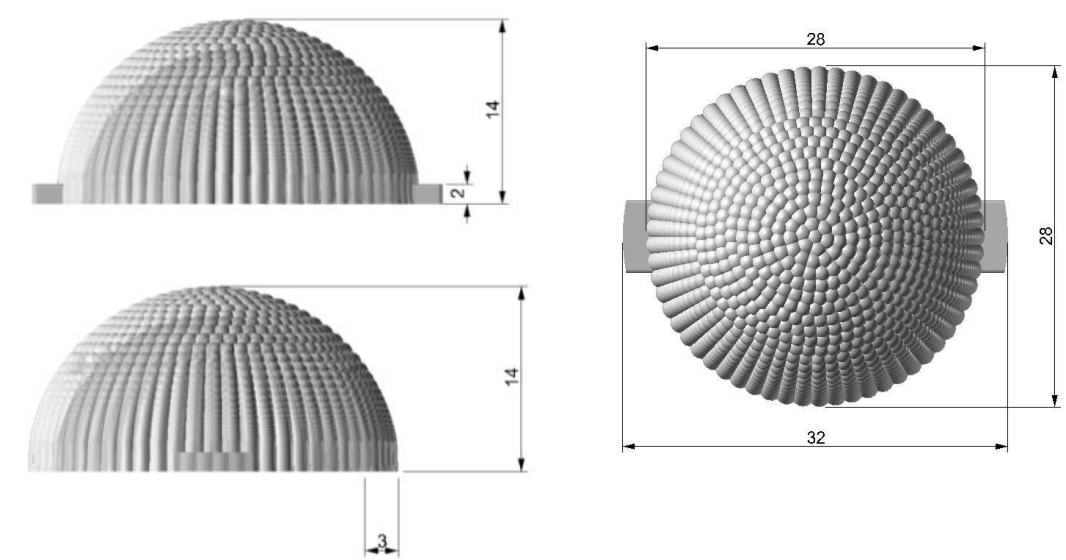

Figure 6 - Design prepared for production, the dimensions are in $\mathrm{mm}$

In order to verify the performance of this shell mixer, we tested it in two different situations: combined with a perfect imaging lens and combined with a parabolic reflector.

The first case to be analyzed by ray tracing was the combination of a RGBW LED with a refractive "super lens" with an ultra-high refractive index $n=5$. This allows the lens to collect a significant amount of light emitted by the source up to $60 \mathrm{deg}$ off axis (the lens was 90 times wider than the mixer). This is a worst case scenario and two light sources were tested: the bare LED and the LED with the shell mixer on top. Figure 7 shows the setup and the results of these two tests. At the bottom left corner we have the image on the target when the light source is the bare LED. At the bottom right corner we have the image on the target when the light source is the LED combined with the shell mixer. As can be seen, in the first case, the "super lens" forms a perfect image of the LED with the four chips of different colors perfectly visible. However, in the second case, the image on the target is a white disk. The internal structure of the light source was lost and the result is white light.

It is very important to notice that the size of the white disk obtained with the shell mixer is about the same as the image of the bare LED. This is an indication of the fact that the shell mixer does not significantly increase etendue. The consequence of this is that the same size optics that are used to control the bare LED emission can also be used to control the emission of the shell mixer. 


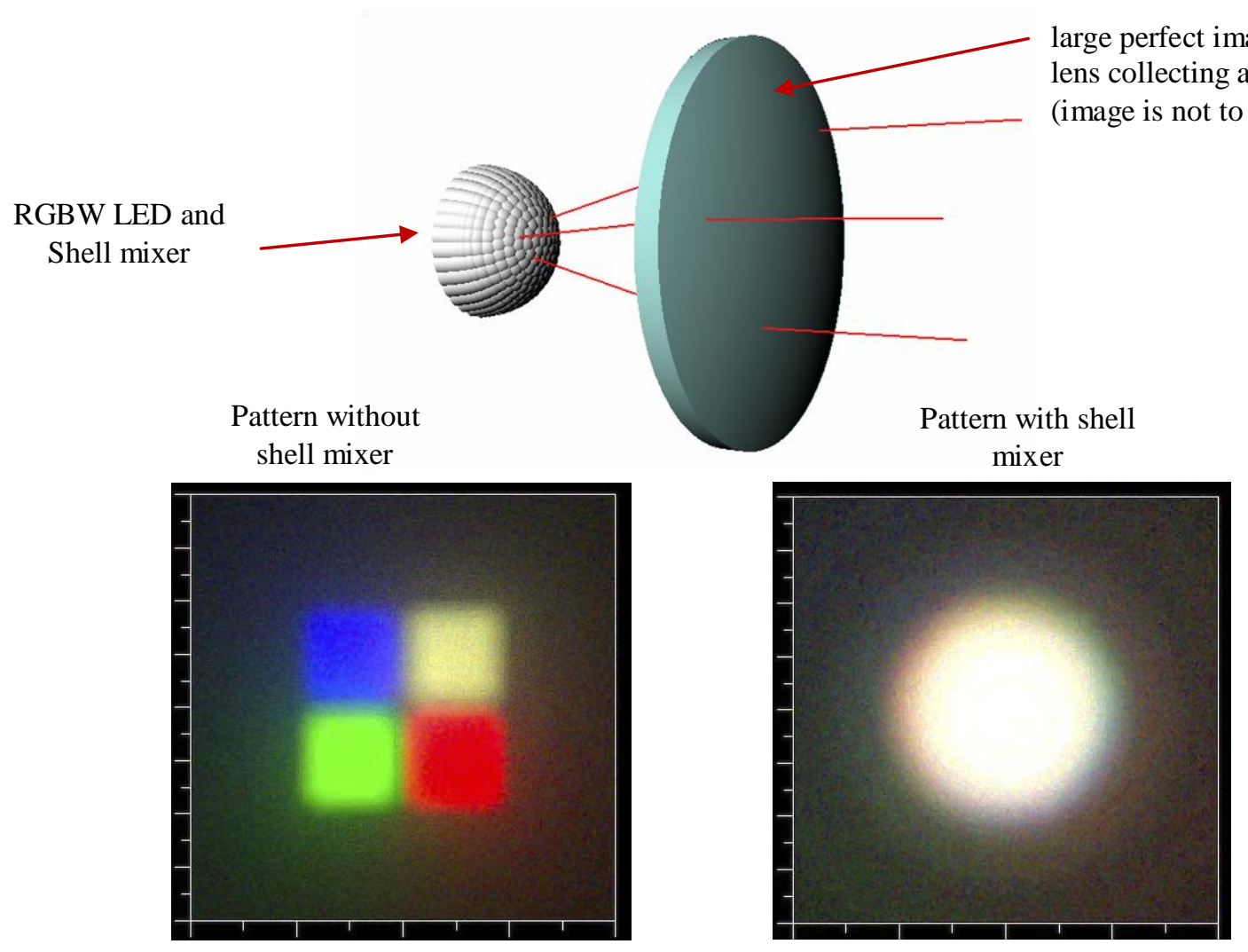

Figure 7 - Top: setup for testing the shell mixer using a lens. Bottom left: colored far-field image of the bare LED produced by a lens imaging the source. Bottom right: same, but with shell mixer on top of the LED.

Another situation which was also analyzed is shown in Figure 8. This situation is more like a typical luminaire. In this test we used an RGBW LED (commercially available and similar to Cree XLamp MC-E Color) and a parabolic reflector. This set was ray traced with and without shell mixer. A sensor was placed $2.5 \mathrm{~m}$ away from the luminarie, the typical distance from ceiling to floor in a house.

Figure 8 at the top shows the overall view of the setup. At the bottom left, we have the pattern on target without the shell mixer and at the bottom right the pattern on target with the shell mixer. 


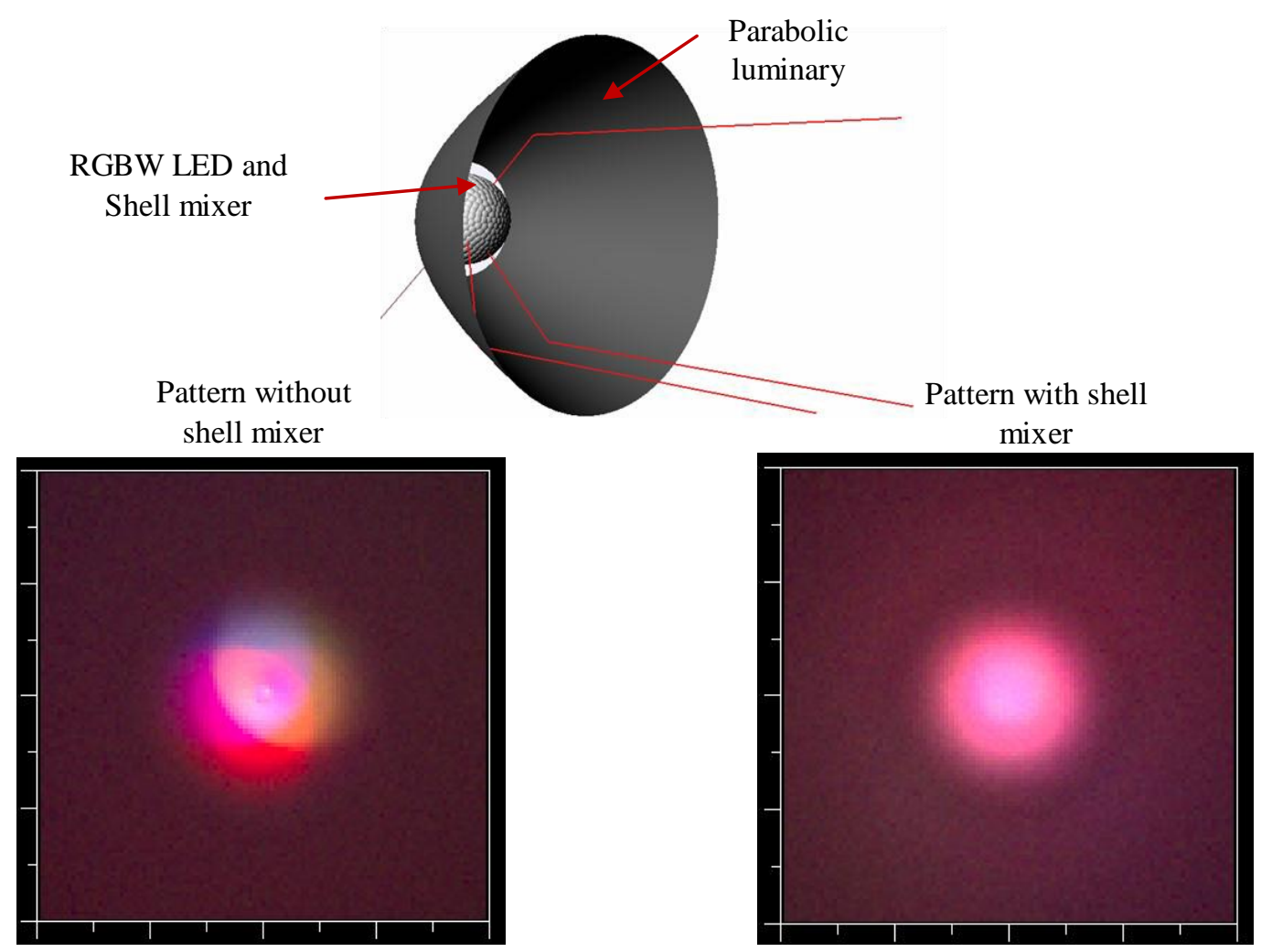

Figure 8 - Top: setup for testing the shell mixer using a collimating reflector. Bottom left: colored far-field image of the bare LED produced by the parabolic mirror. Bottom right: same, but with shell mixer on top of the LED. The sensor plane is placed $2.5 \mathrm{~m}$ away from the luminarie exit plane.

Again, the improvement in the pattern is quite notorious when the shell mixer is added onto the LED.

\section{PROTOTYPE CHARACTERIZATION}

The first shell mixer prototype was made of Ultra-clear Polycarbonate Makrolon LED 2245 by injection molding. It was designed for a chip diameter of $9 \mathrm{~mm}$ and its size was adjusted to fit into a housing that meets the Zhaga standards [6]. Figure 9 shows two pictures of whole shell mixer, when seen from below and from above. 

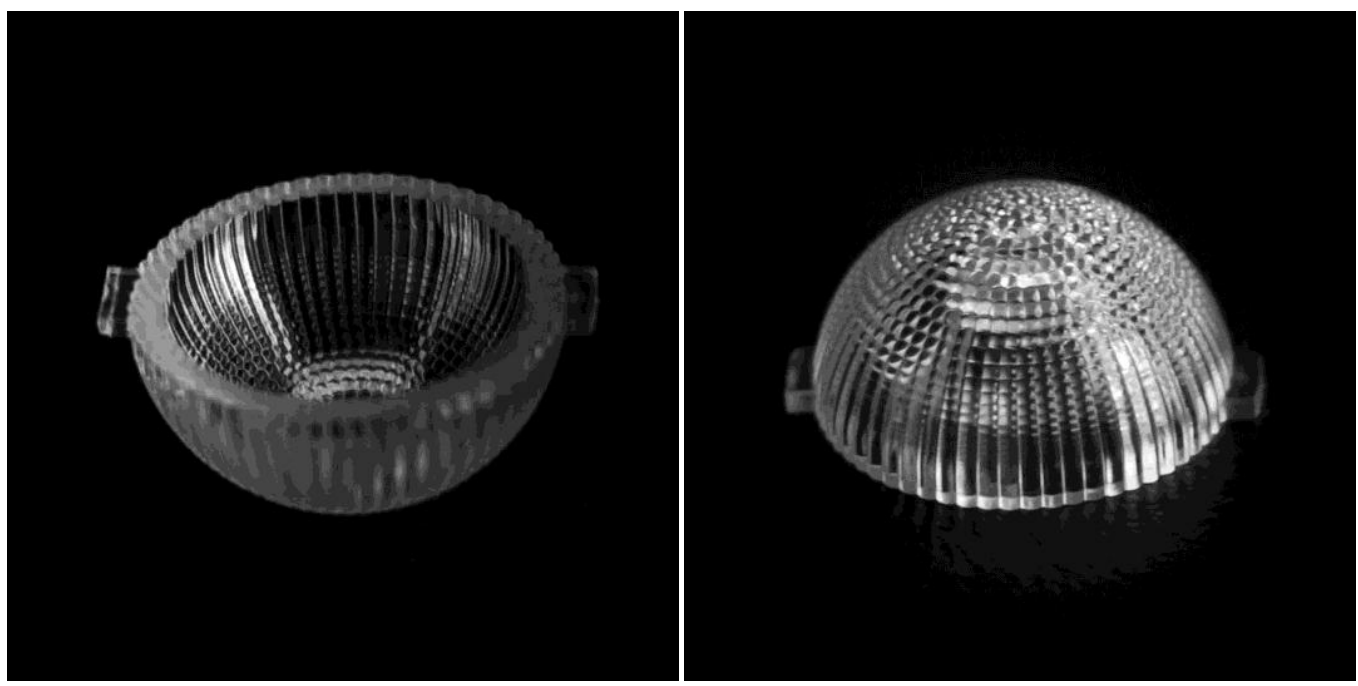

Figure 9 - First shell mixer prototype

Figure 10 shows some details of the shell mixer optic. The left image shows the lenses at the center of the mixer while the image to the right shows the lenses at its edges. These photos were taken under a microscope for surface quality control.
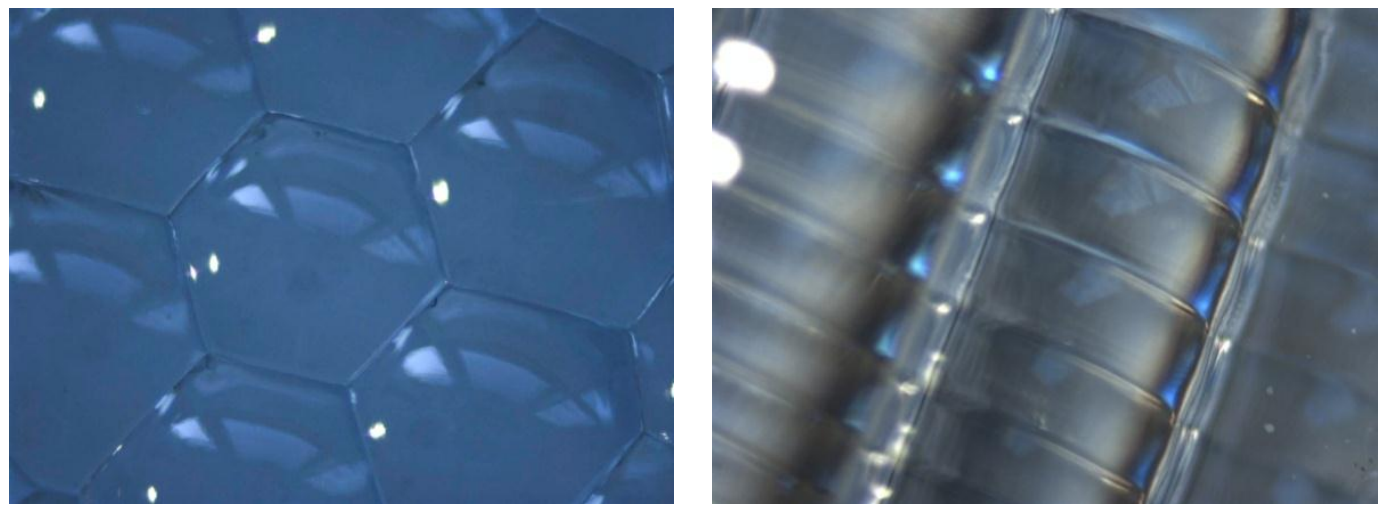

Figure 10 - Left: shell mixer outer lenses at the center of the optic. Right: shell mixer outer lenses at the edge of the optic.

\subsection{LOR (Light Output Ratio)}

The efficiency of the shell mixer can be defined as the light flux exiting the optics that is collectable by a luminaire (within an angular range of $\pm 90 \mathrm{deg}$, for instance) divided by the light flux available at the exit of the LED package. Ray tracing models show that this efficiency or light output ratio (LOR) is very high. This is due, in part, to the recirculation of light between the shell mixer and the LED package. Part of the LED light reaching the shell mixer is reflected back due to Fresnel reflections on the inner surface of the mixer. However, since the mixer overall shape is a dome over the LED, this light is sent back to the source. The high reflectivity of the dies and LED packages (which are white "Lambertian" in the gaps between chips) sends this light back to the shell mixer for another chance to be extracted. This increases the light extraction efficiency.

The measurement of the LOR may be done using an integrating sphere or a goniometer. In both cases we should compare the emission of the bare LED with that with the shell. In the measurements the alignment between shell and the chip was not well controlled, due to the lack of housing. Figure 11 shows a diagram of the setup when the LOR is measured with a goniometer. The LED (with and without the shell mixer) is mounted on a goniometer that rotates it around a vertical axis. Some distance away there is a luxmeter recording how much light it receives. The luxmeter points at the light source through a narrow black tube to block stray light from reflections on other objects. The LED used in this measurement was the OSRAM multicolor LED module that consists of 10 LEDs (4 red, 1 blue and 5 white). Note that this LED module is not commercial, but and R\&D sample (shown in Figure 15). 


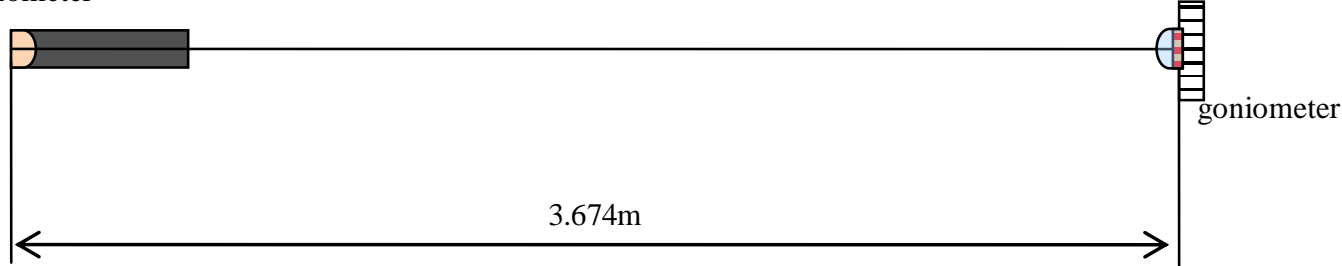

Figure 11 - Schematic representation of efficiency measurement set-up. Note that shell mixer is not assembled on a housing and, therefore, no back-reflected light is being recollected.

This method was used to measure a cross section of the intensity pattern and the result is shown in Figure 12. Since the pattern has rotational symmetry, the overall performance may be obtained from this measurement. Integrating the areas (flux) in the graphs bellow the LED curve and the LED with shell mixer curve results in an efficiency of $89.2 \%$.

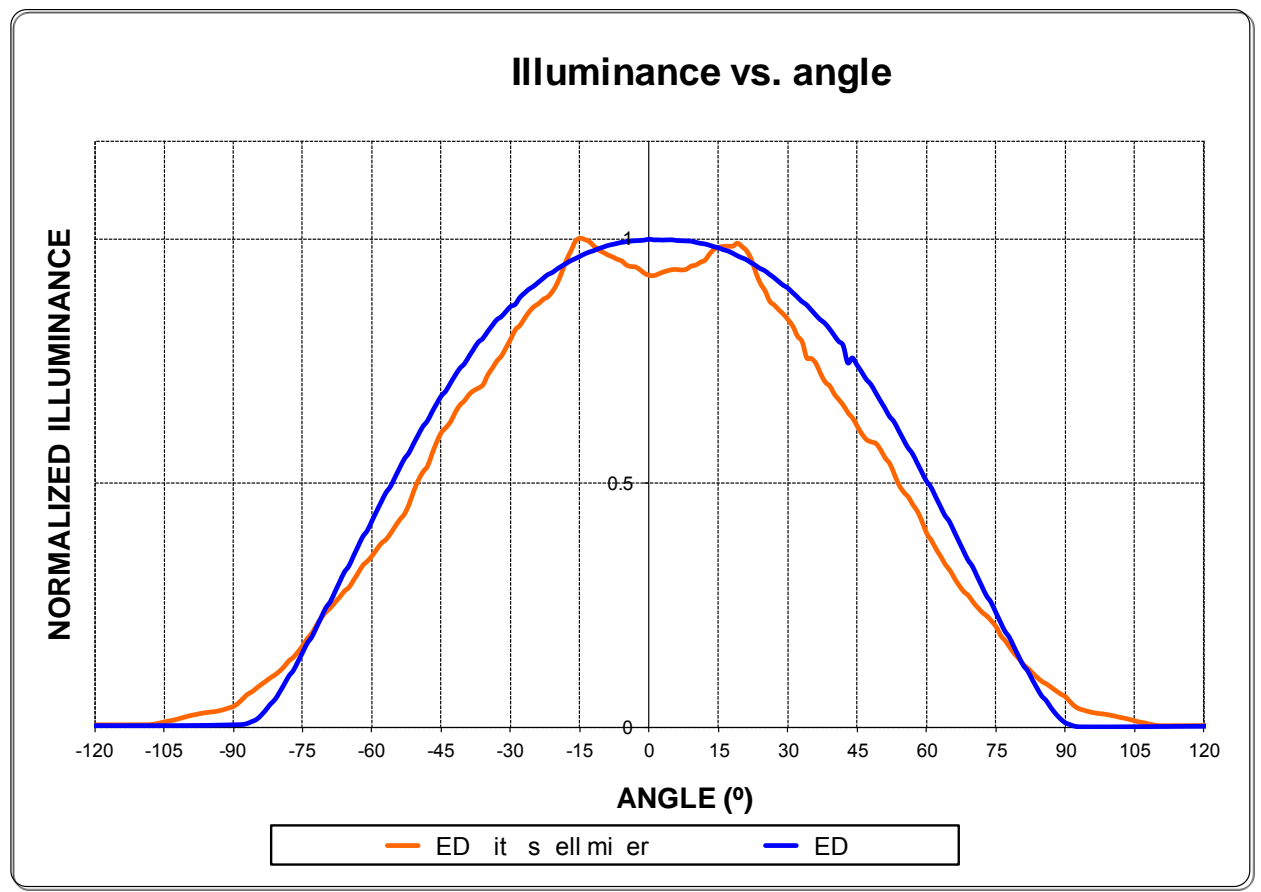

Figure 12 - Illuminance vs. angle for the LED with and without the shell mixer prototype on top of it.

The shell mixer performance was also analyzed with a high efficacy LED module provided by Osram whose package has been designed to achieve high reflectivity. The efficiency of the shell with that LED was measured by Osram in an integrating sphere, resulting in 95\%, which confirms the potential of the shell to recycle part of Fresnel reflections.

Figure 13 shows the shell mixer on top of a multicolor OSRAM R\&D LED module $(7 \mathrm{~mm}$ diameter source consisting of 10 chips: 4 red, 5 white and 1 blue. Instead of 10 different colored sources, one can only see a virtual white source. Note that the image does not change for different viewing angles. From all directions the source appears white and about the same apparent size. 

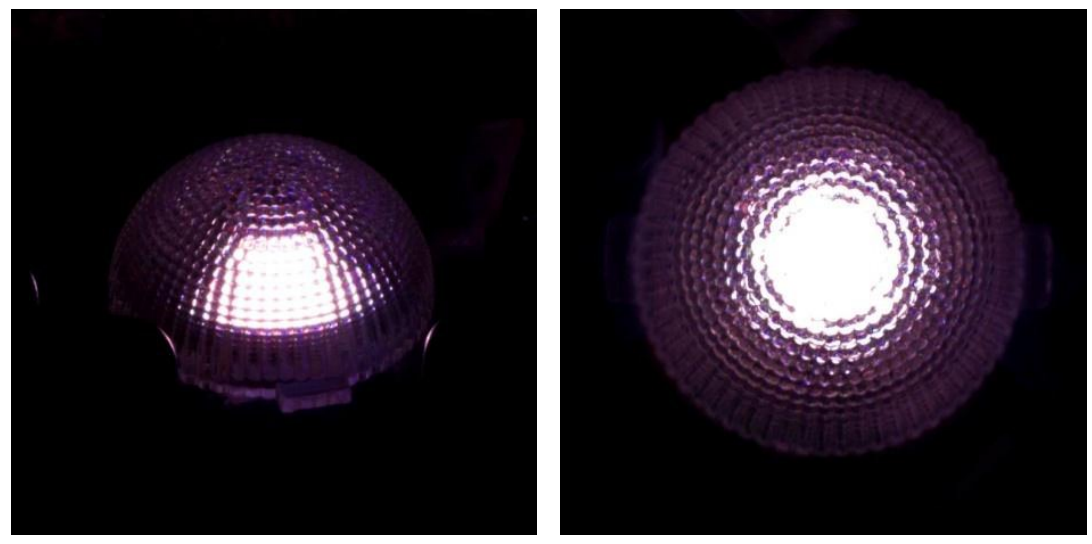

Figure 13 - Shell mixer on top of an OSRAM R\&D multicolor LED module ( $7 \mathrm{~mm}$ diameter source consisting of 10 chips: 5 white, 4 red and 1 blue). Note that, instead of 10 colored chips, only a white virtual source is visible.

\subsection{Illumination pattern}

Figure 14 shows a set-up and images on the wall of an OSRAM multicolour LED module with and without the shell mixer. For this test we have used a low depth of field lens as luminaire and one additional lens to project the far field performance of the shell onto a screen at a finite distance from the set.

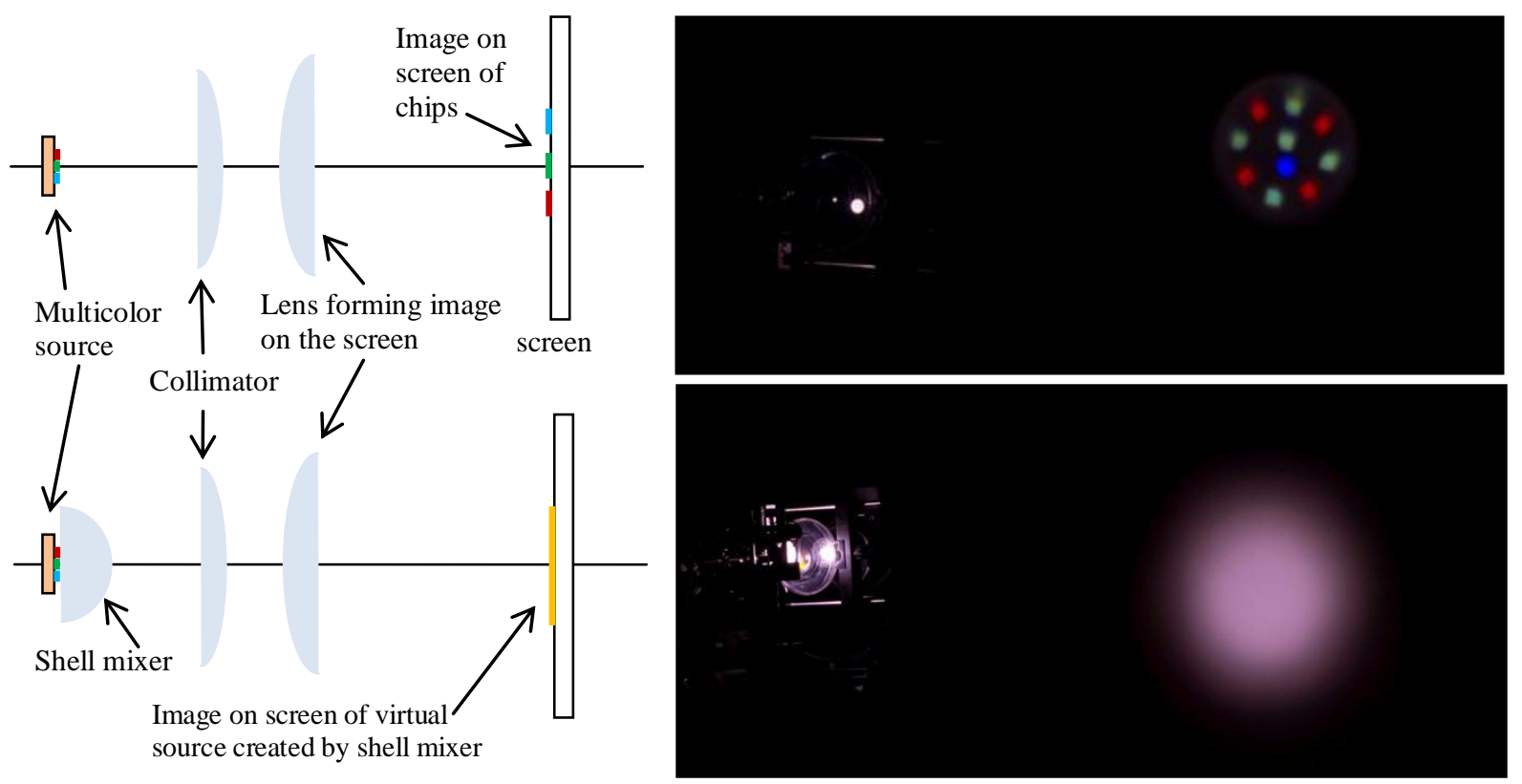

Figure 14 - Schematic representation of the set-up and pictures of the illumination pattern on the wall without (up) and with (down) shell mixer.

Figure 15 shows a different test with a standard parabolic luminaire. Two situations are shown: illumination pattern on the floor (at $2.5 \mathrm{~m}$ from the ceiling) and wall wash. The beam on the floor was tested with an OSRAM LED while the wall wash was tested with a Cree LED. In both cases, adding the shell mixer improves the illuminance uniformity and decreases color artifacts.

These tests were repeated with sources of different diameters to confirm that the etendue increase was constant ( 20\%) for chips smaller than the integration zone area for which the mixer was designed. 


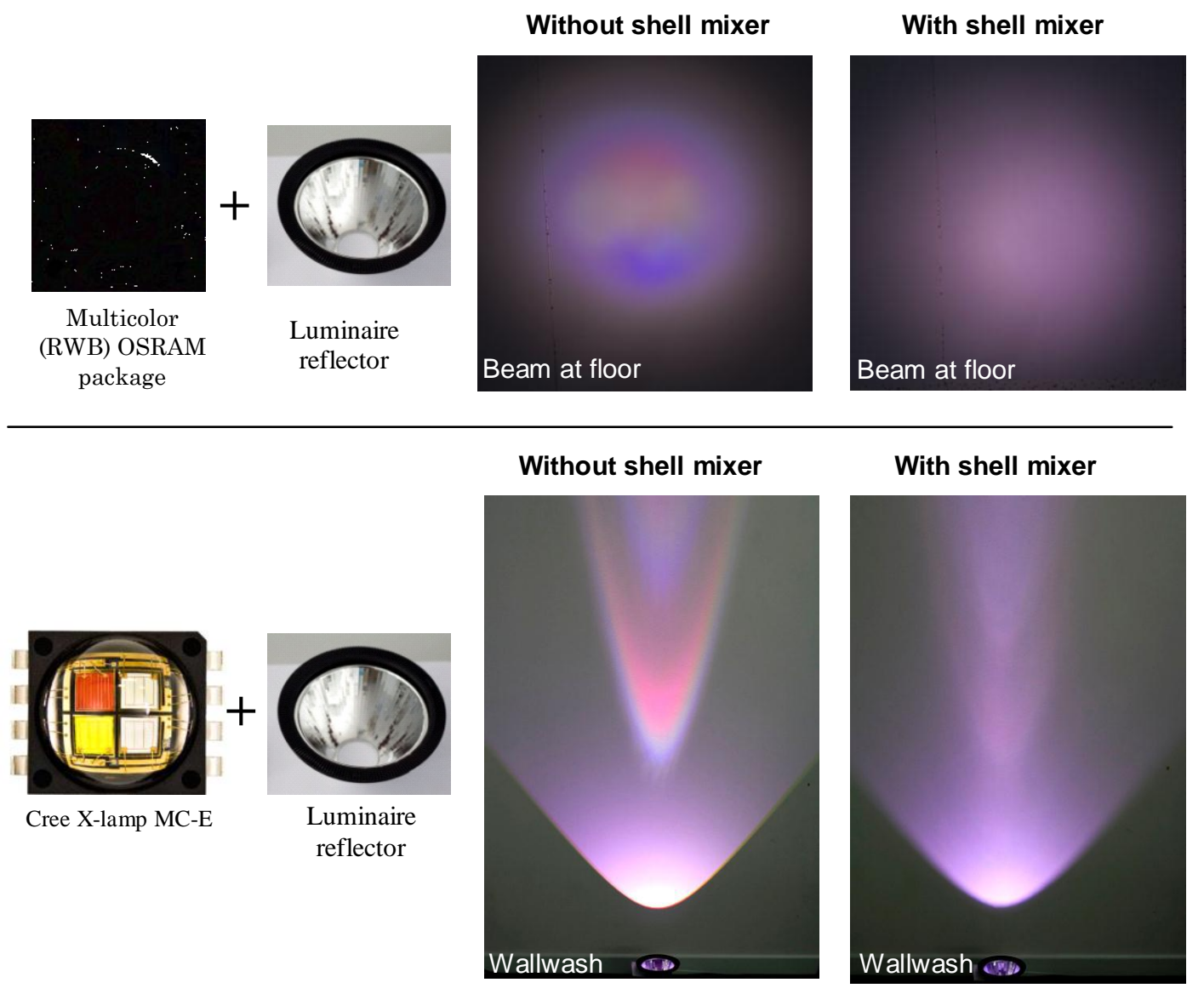

Figure 15 - Performance with and without shell mixer for a luminaire reflector (supplied by Regent lighting) and 2 different LED modules. The beam aesthetics substantially improves with the addition of shell mixer, the effect being more outstanding at the floor for the OSRAM package and in wall wash for the Cree package.

\section{CONCLUSIONS}

The shell-mixer eliminates all problems noticed in conventional lamps, like color shadows, multiple shadows, color fringes and shifts, and any other kind of intensity artifacts from source. The source simply behaves like a single uniform emitting disk, no matter what the real source looks like.

The apparent size of the source increases only slightly (typically by $20 \%$ ), so that the etendue of the source is not increased by much. This is extremely beneficial for high collimation applications like spot lights.

In contrast, if one wants to achieve similar color mixing and smoothing with diffusers, no matter where in the optical path they are used, the apparent source size and the collimation angle increases by a factor of typically 2-3, while the efficiency is compromised due to back scattering losses.

The first shell mixer prototype was built and tested. It shows results close to those obtained by computer simulation. 


\section{ACKNOWLEDGMENTS}

The investigations are conducted as a part of EU and Spanish Government funded projects SSL4EU (Solid state lighting for EU, FP7-257550) and SEM:TSI-020302-2010-65. More information can be found at project website http://ss14.eu. Additional support comes from Spanish Ministry MITYC under projects V-SS, SMS-IMAGING, V-FL and SMS-AUTO (TSI-020100-2011-445, 372, 381 and 444, Plan Avanza 2011), respectively

\section{REFERENCES}

[1] http://ssl4.eu

[2] M. Born, E. Wolf, "Principles of Optics", Pergamon, Oxford (1975)

[3] W. Cassarly, "Nonimaging Optics: Concentration and Illumination", in the Handbook of Optics, $2^{\text {nd }}$ ed., pp2.232.42, McGraw-Hill, New York (2001).Booth, N. and Smith, A. S., [Infrared Detectors], Goodwin House Publishers, New York \& Boston, 241-248 (1997).

[4] O. Dross, R. Mohedano et al. Proc. SPIE 7103, 710306 (2008), DOI: 10.117/12.79811

[5] J. C. Miñano, M. Hernández, P. Benítez, J. Blen, O. Dross, R. Mohedano, A. Santamaría, "Free-form integrator array optics", in Nonimaging Optics and Efficient Illumination Systems II, SPIE Proc., R. Winston \& T.J. Koshel ed. (2005)

[6] http://www.zhagastandard.org/ 